INPLASY

PROTOCOL

To cite: Song et al. Platelet-rich plasma injections in the treatment of tendinopathy: A systematic review with network meta-analyses. Inplasy protocol 202090024. doi:

10.37766/inplasy2020.9.0024

Received: 06 September 2020

Published: 06 September 2020

Corresponding author:

Yinghui Hua

hua_cosm@aliyun.com

Author Affiliation:

Department of Sports

Medicine, Huashan Hospital,

Shanghai, China.

Support: NNS Found of China.

Review Stage at time of this submission: The review has not yet started.

Conflicts of interest:

None.

\section{Platelet-rich plasma injections in the treatment of tendinopathy: A systematic review with network meta-analyses}

Song, Y1; Xue, X2; Li, Q3; Hua, Y4.

Review question / Objective: Which kind of Platelet-rich plasma injections is the best option for the treatment of tendinopathy?

Condition being studied: Tendinopathy is one of the most common cause of pain in sports medicine, which affects the athletes and general populations. Though the epidemiology of tendinopathy is hard to evaluate, it has been reported that around $30 \%$ of consultations with a general practitioner for a musculoskeletal pain complaint are related to tendinopathy. Alone the United States of America, economic burden of musculoskeletal disease has cost US $\$ 796.3$ billion in 20092011 for health care. To optimize treatment of this timeconsuming and costly disorder is challenging but socioeconomically demanded.

INPLASY registration number: This protocol was registered with the International Platform of Registered Systematic Review and Meta-Analysis Protocols (INPLASY) on 06 September 2020 and was last updated on 06 September 2020 (registration number INPLASY202090024).
Rationale: The applications of PRP in the studies of tendinopathy were in conflicts, and the results of the effects of PRP on different kinds of tendinopathy also lacked exploration.

Condition being studied: Tendinopathy is one of the most common cause of pain in sports medicine, which affects the athletes and general populations. Though the epidemiology of tendinopathy is hard to evaluate, it has been reported that around 
$30 \%$ of consultations with a general practitioner for a musculoskeletal pain complaint are related to tendinopathy. Alone the United States of America, economic burden of musculoskeletal disease has cost US $\$ 796.3$ billion in 20092011 for health care. To optimize treatment of this time-consuming and costly disorder is challenging but socioeconomically demanded.

\section{METHODS}

Search strategy: We searched PubMed, Web of Science, Embase, and Cochrane Library up to 6 September 2020. There were no restrictions on language or publication date. We also reviewed the reference lists of included publications and relevant reviews to identify any additional studies that were missed in the electronic database. The search was based on the following keywords: tendinopathy, tendinosis, tendinitis, and tendon injury with the limitation of randomized controlled trials (RCTs).

Participant or population: Adult patients (age > 18 years) who suffered from tendinopathy but not caused by significant trauma or other systemic diseases.

Intervention: Eligible interventions included injections of any autologous blood product including whole blood, PRP or platelet-poor plasma (PPP), or autologous conditioned plasma (ACP).

Comparator: Controls were accepted as dry needling, saline, local anesthetic, placebo (PLA) or a 'wait and see' strategy.

\section{Study designs to be included: RCT.}

Eligibility criteria: We included randomised controlled trials (RCTs) that used PRP or autologous blood for tendinopathy of any type with a follow-up of at least 2 weeks. We considered both the two-arm parallel group and multi-arm trial designs. Adult patients (age $>18$ years) who suffered from tendinopathy but not caused by significant trauma or other systemic diseases were eligible for inclusion in this systematic review and network meta-analysis. We excluded animal studies, patients undergoing surgery, results reported as neither mean $\pm S D$ nor other forms which could be converted to it, and controls as corticosteroid, eccentric training, shock wave treatment. Eligible interventions included injections of any autologous blood product including whole blood, PRP or platelet-poor plasma (PPP), or autologous conditioned plasma (ACP). Controls were accepted as dry needling, saline, local anesthetic, placebo (PLA) or a 'wait and see' strategy.

Information sources: We searched PubMed, Web of Science, Embase, and Cochrane Library up to 6 September 2020. There were no restrictions on language or publication date. We also reviewed the reference lists of included publications and relevant reviews to identify any additional studies that were missed in the electronic database.

Main outcome(s): The primary outcome of this study was pain relief since pain is the predominant symptom in patients with different kinds of tendinopathy. For all study outcomes, there were two follow-up periods of assessment: 1. Short term: up to 3 months after treatment. 2. Intermediate and mediate term: more than 3 months after treatment.

Additional outcome(s): The secondary outcome was functional improvement. Since functional scales vary in different sides, the most frequently used measurements in each tendinopathy were summarized.

Data management: Data extraction was independently performed by two researchers ( $Y S$ and $X X$ ), and included the following elements: authors, year of publication, number of the patients allocated to intervention and control groups, mean age, mean follow-up time, and outcome measures (pain relief or functional recovery) and the kind of tendinopathy (e.g. patellar or achilles tendinitis). If numeric outcome data were unavailable, we extracted the data from 
figures and graphs. In cases with missing information, the study authors were contacted to request their data.

Quality assessment / Risk of bias analysis: Two researchers (YS and XX) independently assessed the quality of all included RCTs using the Cochrane risk of bias. The following domains were considered: random sequence generation, allocation concealment, blinding of participants and outcome assessors, incomplete outcome data, selective reporting and other bias. Each entry was classified to be at a low, unclear or high risk of bias. The inter-rater agreements of the initial ratings were calculated, and the third reviewer (YH) would be consulted for disagreements.

Strategy of data synthesis: In this study, we performed two types of meta-analyses in each kind of tendinopathy. For all study outcomes, we conducted pairwise metaanalyses to get the direct estimates for each included comparison. Then we proceeded with network meta-analysis to get the indirect estimates. We conducted pairwise meta-analysis of change-frombaseline scores using a random-effects model. Statistical heterogeneity was quantified with the 12 statistic. A p value of less than 0.05 was judged to be statistically significant. Then we integrated direct comparison evidence and constructed network meta-analysis of change scores with frequentist methods. A random-effects model was also used to analyze the efficacy of all of the treatments simultaneously. The Surface Under the Cumulative RAnking (SUCRA) method was used. The SUCRA value was presented as the percentage of the area under the curve. It ranged from $0 \%$ to $100 \%$, where $100 \%$ reflected the best treatment effect, and $0 \%$ reflected the worst treatment effect without any uncertainty. The inconsistency analysis was assessed using the node-splitting method, which concerned the difference between the results from direct and indirect comparisons ( $P$ O 0.8 as almost perfect agreement. All statistical analyses were conducted using STATA software (V.13.0, Stata, College Station, Texas, USA) and $\mathbf{R}$ (V.3.6.1, R Foundation for Statistical Computing, Vienna, Austria).

Subgroup analysis: All meta-analyses were performed in each kind of tendinopathy (e.g. patellar or achilles tendinitis).

Sensibility analysis: None.

Language: English.

Country(ies) involved: China.

Other relevant information: None.

Keywords: Platelet-rich plasma; tendinopathy.

Dissemination plans: Published in SCI Journals.

Contributions of each author:

Author 1 - Yujie Song - Study design; literature search and selection; data collection; quality rating; writing of the manuscript.

Author 2 - Xiao'ao Xue - Study design; literature search and selection; data collection; quality rating; statistical analysis; reviewing of the manuscript.

Author 3 - Qianru Li - Study design; reviewing the manuscript.

Author 4 - Yinghui Hua - Study design; supervision of literature search, data collection and quality rating; reviewing the manuscript. 